\title{
Review: patients living with diabetes mellitus focus on learning to balance by assuming control for the management of their illness
}

\author{
Paterson BL, Thorne S, Dewis M. Adapting to and managing diabetes. Image J Nurs Sch 1998 First Quarter;30:57-62.
}

\section{Question}

What is the "lived experience" of people who have diabetes mellitus?

\section{Data sources}

Unpublished and published studies (including dissertations, theses, articles, and book chapters) were identified by searching 6 databases (Sociofile, PsycLit, Dissertation Abstracts, CINAHL, Medline, and Allied Health); the Canadian Nurses Association library; and refereed nursing, social science, and allied health journals and books published from 1980 to July 1996.

\section{Study selection}

Studies were included if they were qualitative interpretive research reports of the experience of living with diabetes from the perspective of an insider and included participants with diagnosed diabetes.

\section{Data analysis}

Data were analysed using meta-ethnographic synthesis (inductive analysis of data from individual studies, hypothesis development, and testing of hypothetical relations). Analyses of individual studies were reviewed by multiple researchers.

\section{Main results}

43 reports of 38 studies were identified ( 6 phenomenological, 5 ethnographic, 2 case, 2 focus group, 9 exploratory, and 19 grounded theory study reports). Most reports included only participants who were white $(n=33)$ or who had type 1 (insulin dependent) diabetes mellitus $(\mathrm{n}=33)$. Participants were usually married women who had a minimum high school education.

Patient experiences of adapting to and managing diabetes are captured by the concept of balance. Patients want to achieve a balance between living with diabetes and the desire for a normal healthy life. Learning to balance involves making the decision to assume control and then actually assuming control. Patients vary in the degree to which they choose to assume control of diabetes management and revise this in response to certain life events (eg, depressive episodes).
Assuming control involves knowing one's body, learning how to manage diabetes, and fostering supportive, collaborative relationships with others. Knowing the body refers to paying attention to the body's cues that blood sugar levels may be high or low, deciphering what the cues mean, recognising unique patterns of body responses that may cause changes in blood sugar levels, and testing blood sugar levels to validate that cues and hypotheses about causes are correct. Learning how to manage diabetes involves a progression of getting regulated, trying out and modifying a prescribed regimen to fit the circumstances, and establishing a unique basic routine. This progression is repeated each time patients encounter unfamiliar body responses to interventions or situations. Basic knowledge of diabetes pathophysiology and the rationale for common interventions, a belief in one's ability to manage diabetes, and certain life events affect the progression of diabetes management. Adapting to diabetes involves accepting one's self as a "diabetic," experiencing losses and feelings of being different, and reframing complications in a positive light. Fostering supportive, collaborative relationships involves balancing one's need for support and advice with the need to maintain autonomy and self esteem. Recruitment of "allies" who have a basic understanding of diabetes and are familiar with a patient's unique responses is important. Allies, often family members, can serve as rescuers when hypoglycaemia occurs and as secondary monitors of body cues. People with diabetes try to maintain a balance between expressing their own needs and being seen as troublesome in their relationships with caregivers. Helpful healthcare interactions are characterised by caregivers who know and value the patient as an individual.

\section{Conclusion}

The lived experience of patients with diabetes mellitus focuses on learning to balance the management of their disease with the desire for a normal life by making the decision to assume control and then following through.

Source of funding: Canadian Nurses Foundation

For correspondence: $\operatorname{Dr} B$ L Paterson, School of Nursing, University of British Columbia, 2211 Wesbrook Mall, Vancouver, British Columbia V6T 2B5, Canada.Fax +1 6048227466.

\section{Commentary}

Although a reasonable amount of research explores living with diabetes, this study by Paterson et al is unique. The results of qualitative studies were synthesised to provide rich information and valuable new insights about how people with diabetes adapt to and manage their illness.

The findings show that people with diabetes try to balance the demands of living a normal life with managing the condition. This concept of balance has important implications for nursing and is particularly pertinent for those working in hospital and primary care settings. The importance of a collaborative relationship, in which the knowledge and experiences of the person with diabetes are valued, is affirmed. Partnership and individualised care are essential prerequisites for the sharing of information. Nurses have a crucial role in educating individuals with diabetes, but this must be done in the context of helping them to learn from experience how specific knowledge can be integrated into their lives, rather than trying to enforce textbook "rules and regulations." Indeed, as the authors discuss, the notion of compliance becomes obsolete if nurses help people to achieve balance rather than "diabetes control."

The strength of the study lies in its methodology-a meta-ethnography of 38 studies. The limitations, which are acknowledged by the authors, are that most of the participants were well educated, white women with type 1 diabetes. The findings therefore may only be applied with caution to individuals not in this group. However, these limitations reflect the paucity of studies investigating the lived experience of people with type 2 diabetes, those in lower socioeconomic groups, and men. Given that $85 \%$ of people with diabetes have type 2 and nearly half are men, it is clear that further research is urgently needed. ${ }^{1}$

Maggie Watkinson, RN, MSc Lecturer Practitioner in Diabetes Nursing Radcliffe Infirmary NHS Trust and Oxford Brookes University Oxford,UK

1 Bowen Jones D, Gill GV. Non-insulin dependent diabetes mellitus: an overview. In: Pickup J, Williams G, editors. Second edition. Tickup J, diabetes. Oxford: Blackwell Science, 1997. 